\title{
Spin Wave Excitation in Magnetic Insulator Thin Films by Spin-Transfer Torque
}

\author{
Jiang Xiao ${ }^{\mathrm{a}, \mathrm{b}}$, Yan Zhou ${ }^{\mathrm{c}}$, Gerrit E. W. Bauer ${ }^{\mathrm{d}, \mathrm{e}}$ \\ ${ }^{a}$ Department of Physics and State Key Laboratory of Surface Physics, Fudan University, Shanghai, China \\ ${ }^{b}$ Center for Spintronic Devices and Applications, Fudan University, Shanghai, China \\ ${ }^{c}$ Department of Physics, The University of Hong Kong, Hong Kong, China \\ ${ }^{d}$ Institute for Materials Research \& WPI-AIMR, Tohoku University, Sendai 980-8557, Japan \\ ${ }^{e}$ Kavli Institute of NanoScience, Delft University of Technology, 2628 CJ Delft, The Netherlands
}

\begin{abstract}
We describe excitation of dipole-exchange spin waves in insulating magnetic thin films by spin current injection at the surface of the film. An easy-axis magnetic surface anisotropy can induce a non-chiral surface spin wave mode with penetration depth inversely proportional to the strength of the surface anisotropy, which strongly reduces the critical current and enhances the excitation power. The importance of the interface spin wave modes on the excitation spectrum is reduced by spin pumping, which depends on the quality of the interface as expressed by the spin mixing conductance.
\end{abstract}

Keywords:

spin-transfer torque, spin wave, magnetic insulator, surface anisotropy, spin pumping

\section{Contents}

1 Introduction \& background

2 Spin current induced magnetization dynamics 3

3 Dispersion, amplification, and dissipation of spin waves in magnetic insulators 6

3.1 Without surface anisotropy . . . . . . . . . . . . . . .

3.2 With easy-axis surface anisotropy . . . . . . . . . . . . . . . . . 10

3.3 With hard-axis surface anisotropy . . . . . . . . . . . . . . . . . . . 14

3.4 Excitation power spectrum . . . . . . . . . . . . . . . 14

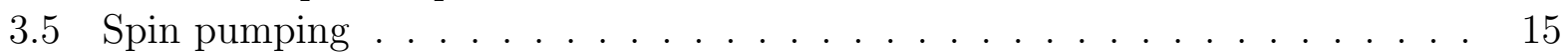

4 Discussion 17

5 Acknowledgment 18

Preprint submitted to Solid State Physics

April 20, 2021 


\section{Introduction \& background}

Since its prediction [1, 2], the spin-transfer torque has been studied extensively both experimentally and theoretically in nano-structures, including magnetic point contacts, spin valves, magnetic tunnel junctions, magnetic thin film structures containing magnetization textures such as magnetic domain walls, vortices, skyrmions, etc. [3] When the torque is sufficiently large at a certain critical current, the magnetization is set into motion. In nanostructures the current-induced dynamics is often well described by the macrospin model that assumes uniform collective motion of the entire magnetization. On the other hand, by employing the (inverse) spin Hall effect [4], a uniform spin current can be injected into a macroscopically large area, thereby possibly exciting the whole spectrum of spin waves.

A spintronic circuit consists of devices that perform operations and interconnects that transport information. Spin information communication over long distances requires weak coupling with the outside world, while spintronic devices require strong coupling with an external control parameter in order to efficiently manipulate the spins. Materials and designs that fulfil these conflicting demands are highly desirable. Spin waves can propagate over much longer distances than diffusive spin currents, in materials such as the magnetic insulator Yttrium-Iron-Garnet (YIG) [5]. Therefore it appears attractive to transmit spin information via spin waves rather than particle-based spin currents [6]. To interface a spintronic circuit, an electrical signal has to be encoded into a spin signal, and spin information has to be read out as a voltage signal. This scenario has been demonstrated by Kajiwara et al. [7] using a Pt-YIG-Pt structure as schematically shown in Fig. 1, in which the encoding is realized by exciting spin waves in a magnetic thin film electrically using the spin Hall effect and spin-transfer torque [1, 2]. The readout is realized by the inverse effect - spin pumping [8], which converts spin waves back into a spin current and via the inverse spin Hall effect, a voltage [7, 9, 10]. The full scenario can be described by six consecutive processes: i) the spin Hall effect in $\mathrm{N}$ generates a spin current $\mathbf{J}_{s}$ polarized long $-\hat{\mathbf{z}}$ by a charge current $J$ flowing in $-\hat{\mathbf{y}}$-direction that is absorbed by the YIG film, ii) the spin current exerts a torque on the magnetization in YIG at the interface, iii) spin wave is excited when the spin-transfer torque overcomes the damping, iv) the excited spin waves propagate to the Pt detector on the right, $\mathrm{v}$ ) the spin wave reaching the second interface contact pumps a spin current into the right $\mathrm{Pt}$, vi) the pumped spin current is converted back into a charge current via the inverse spin Hall effect.

In the experiment, spin waves were excited in a $d=1.3 \mu \mathrm{m}$-thick YIG film by a threshold charge current of $J_{c} \sim 10^{9} \mathrm{~A} / \mathrm{m}^{2}$ in a $10 \mathrm{~nm}$ thick Pt overlayer [7]. This value is much less than expected for a volume spin wave excitation that in the macrospin model is estimated to be $J_{c}=\left(1 / \theta_{H}\right) e \alpha \omega M_{s} d / \gamma \hbar \sim 5 \times 10^{11} \mathrm{~A} / \mathrm{m}^{2}$, where $e$ and $\gamma=e / 2 m$ are the electron charge and gyromagnetic ratio, respectively, and we used the material parameters in Table 1 for the ferromagnetic resonance frequency $\omega$, the spin Hall angle $\theta_{H}$, magnetic damping $\alpha$, and saturation magnetization $M_{s}$. 


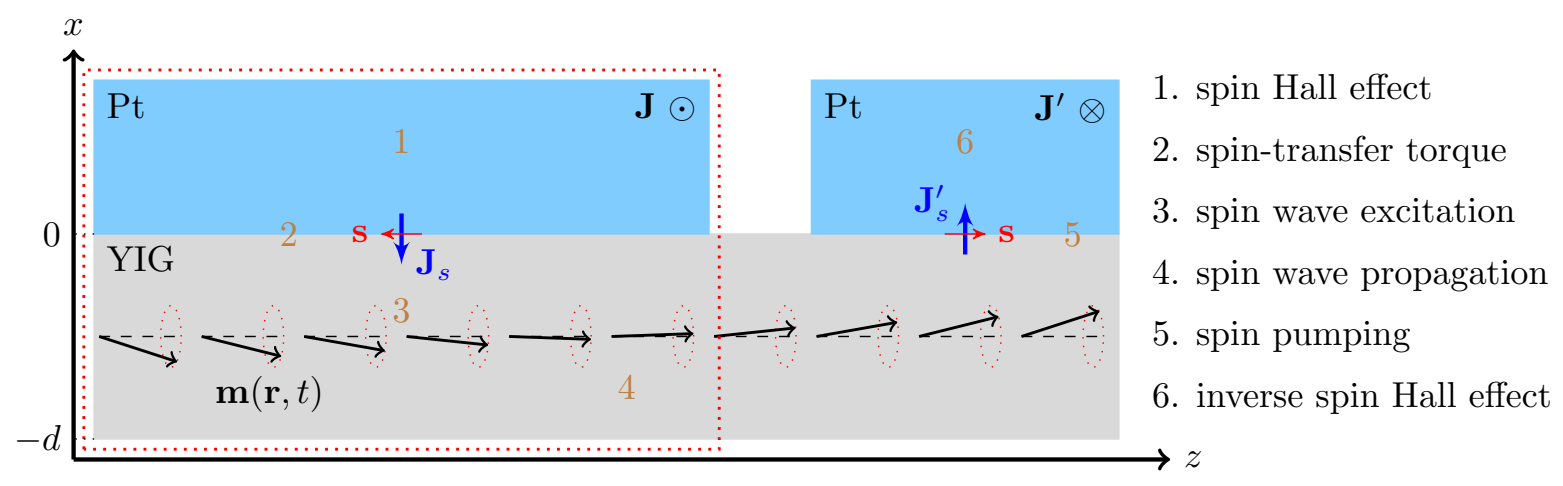

Figure 1: Schematic side view of the experimental setup (Ref. [7]). The six consecutive processes: 1) the spin Hall effect generates a spin current in the Pt on the left, 2) the spin current exerts a spin-transfer torque on the magnetization at the interface, 3) spin wave is excited in YIG when the spin-transfer torque overcomes the damping, 4) the excited spin wave propagates within the film, 5) the spin wave pumps a spin current into the $\mathrm{Pt}$ contact on the right, 6) the inverse spin Hall effect converts the pumped spin current into a charge current.

In this Chapter, we focus on the excitation part, i.e. the processes inside the dashed box in Fig. 1. We describe the spin wave dispersion and excitation in insulating magnetic thin films by the spin-transfer torque in the linear response regime, obtaining the threshold currents for excitation of various spin wave modes and the low-excitation power spectrum.

\section{Spin current induced magnetization dynamics}

We study the spin wave excitation in a bilayer structure as shown in the dotted box region in Fig. 1, in which a non-magnetic $(\mathrm{N})$ metallic thin film is in contact with a ferromagnetic insulator (FI) with equilibrium magnetization along the $\hat{\mathbf{z}}$-direction. The charge current in Pt flows normal the displayed cross section.

The spatial dependent dynamics of the magnetization unit vector $\mathbf{m}(\mathbf{r}, t)$ within the ferromagnetic film is described by the Landau-Lifshitz-Gilbert-Slonczewski (LLGS) equation:

$$
\dot{\mathbf{m}}=-\gamma \mathbf{m} \times\left[\mathbf{H}_{0}+\mathbf{H}_{s}+\frac{A_{\mathrm{ex}}}{\gamma} \nabla^{2} \mathbf{m}+\mathbf{h}\right]+\alpha \mathbf{m} \times \dot{\mathbf{m}}+\frac{\gamma}{M_{s}}\left(\boldsymbol{\tau}_{\mathrm{STT}}+\boldsymbol{\tau}_{\mathrm{SP}}\right),
$$

where $\mathbf{H}_{0}$ includes the external and internal magnetic field, $\mathbf{H}_{s}$ is the surface anisotropy field, $A_{\text {ex }}$ is the exchange constant, $\mathbf{h}(\mathbf{r}, t)$ is the dipolar magnetic field due to $\mathbf{m}(\mathbf{r}, t), \alpha$ is the Gilbert damping constant, $\boldsymbol{\tau}_{\mathrm{STT}}$ is the spin-transfer torque due to the spin current injection from the top surface and $\boldsymbol{\tau}_{\mathrm{SP}}$ is the spin pumping torque due to the magnetization dynamics at the interface.

Due to the broken symmetry, the spins at the surface experience a modified magnetic anisotropy that is usually uniaxial and can be described by a surface anisotropy field [11]:

$$
\mathbf{H}_{s}=\frac{2 K_{1}}{M_{\mathfrak{s}}}(\mathbf{m} \cdot \mathbf{n}) \mathbf{n},
$$


where $\mathbf{n}$ is the outward normal as seen from the ferromagnet which can be the easy or hard axis, depending on the sign of the anisotropy constant $K_{1}$.

At the top surface $(x=0$, see Fig. 1), a spin Hall effect induced spin current density $\mathbf{J}_{s}=J_{s} \hat{\mathbf{z}}$ polarized along $\pm \hat{\mathbf{z}}$ flowing in $-\hat{\mathbf{x}}$ direction gives rise to a spin accumulation in $\mathrm{N}$, which cancels out the longitudinal component of the spin current $\mathbf{J}_{s}$ parallel to $\mathbf{m}$. The transverse component is absorbed at the surface as spin-transfer torque, which drives the magnetization dynamics [12]. The spin pumping exerts a torque $\boldsymbol{\tau}_{\mathrm{SP}}$ on $\mathbf{m}$. The volume density of the spin transfer torque and spin pumping torque can be written as

$$
\boldsymbol{\tau}_{\mathrm{STT}}=J_{s} \mathbf{m} \times \hat{\mathbf{z}} \times \mathbf{m} \delta(x) \quad \text { and } \quad \boldsymbol{\tau}_{\mathrm{SP}}=\hbar \frac{g_{r}}{4 \pi} \hat{\mathbf{z}} \times \dot{\mathbf{m}} \delta(x)
$$

where $g_{r}$ is the real part of the mixing conductance per area for the Pt/YIG interface and the $\delta$-function indicates that both torques are localized at the surface. There is no currentinduced torque inside the bulk because the magnet is assumed to be electrically insulating.

Assuming that the bottom surface $(x=-d)$ is free from the effects of spin-transfer torque, spin pumping, and surface anisotropy, by integrating Eq. (1) over a volume across the surface $x \in\left[0^{-}, 0^{+}\right]$and $x \in\left[-d^{-},-d^{+}\right]$, we obtain the boundary conditions for $\mathbf{m}$ at $x=0$ and $-d[1]$ :

$$
\begin{aligned}
A_{\mathrm{ex}} \mathbf{m} \times \frac{\partial \mathbf{m}}{\partial \mathbf{n}}-\frac{2 \gamma K_{s}}{M_{s}}(\mathbf{m} \cdot \mathbf{n}) \mathbf{m} \times \mathbf{n}+\frac{\gamma J_{s}}{M_{s}} \mathbf{m} \times \hat{\mathbf{z}} \times \mathbf{m}+\frac{\gamma \hbar g_{r}}{4 \pi M_{s}} \hat{\mathbf{z}} \times \dot{\mathbf{m}}=0 \quad \text { at } \quad x=0, \\
A_{\mathrm{ex}} \mathbf{m} \times \frac{\partial \mathbf{m}}{\partial \mathbf{n}}=0 \quad \text { at } \quad x=-d,
\end{aligned}
$$

with $\partial \mathbf{m} / \partial \mathbf{n} \equiv(\mathbf{n} \cdot \nabla) \mathbf{m}$ and $K_{s}=\int_{0^{-}}^{0^{+}} K_{1} d x$. We convert surface anisotropy, spin current, and spin pumping parameters into effective wave numbers by defining:

$$
k_{s}=\frac{2 \gamma K_{s}}{A_{\mathrm{ex}} M_{s}}, \quad k_{j}=\frac{\gamma J_{s}}{A_{\mathrm{ex}} M_{s}}, \quad \text { and } \quad k_{p}=\frac{\gamma g_{r} \hbar \omega_{0}}{4 \pi A_{e x} M_{s}} .
$$

As we assumed complete absorption of the transverse spin current at the interface, $k_{j}$ literally means the absorbed spin current. Analogously, the wave number $k_{c}=\alpha\left(\omega_{0}+\omega_{M} / 2\right) d / A_{\mathrm{ex}}$ corresponds to the threshold current for exciting the uniform mode of thickness $d$.

The bulk magnetization inside the film satisfies the LLG equation:

$$
\dot{\mathbf{m}}=-\gamma \mathbf{m} \times\left[\mathbf{H}_{0}+\frac{A_{\mathrm{ex}}}{\gamma} \nabla^{2} \mathbf{m}+\mathbf{h}\right]+\alpha \mathbf{m} \times \dot{\mathbf{m}} \quad \text { for } \quad-d<x<0,
$$

where the dipolar magnetic field $\mathbf{h}(\mathbf{r}, t)$ satisfies Maxwell's equations in the quasistatic approximation:

$$
\begin{aligned}
\nabla \times \mathbf{h}(\mathbf{r}, t) & =0, \\
\nabla \cdot \mathbf{b}(\mathbf{r}, t)=\nabla \cdot\left[\mathbf{h}(\mathbf{r}, t)+\mu_{0} M_{s} \mathbf{m}(\mathbf{r}, t)\right] & =0 \quad \text { for } \quad-d \leq x \leq 0, \\
\nabla \cdot \mathbf{b}(\mathbf{r}, t)=\nabla \cdot \mathbf{h}(\mathbf{r}, t) & =0 \quad \text { for } \quad x<-d \quad \text { or } \quad x>0,
\end{aligned}
$$


with boundary conditions

$$
\begin{array}{cl}
\mathbf{h}_{y, z}\left(0^{-}\right)=\mathbf{h}_{y, z}\left(0^{+}\right), & \mathbf{b}_{x}\left(0^{-}\right)=\mathbf{b}_{x}\left(0^{+}\right) \\
\mathbf{h}_{y, z}\left(-d^{-}\right)=\mathbf{h}_{y, z}\left(-d^{+}\right), & \mathbf{b}_{x}\left(-d^{-}\right)=\mathbf{b}_{x}\left(-d^{+}\right) .
\end{array}
$$

Eqs. (4- 8) completely describe what is called dipolar-exchange spin waves. The method described above extends previous studies by De Wames and Wolfram [13] and Hillebrands [14] by including the current-induced spin-transfer torque, spin pumping, and surface anisotropy.

In general, the non-linear LLG equation in Eq. (6) can not be solved easily. However, focussing on small deviations of $\mathbf{m}$ from its equilibrium direction ( $\hat{\mathbf{z}}$-direction), i.e. $\mathbf{m}(\mathbf{r}, t)=$ $m_{z} \hat{\mathbf{z}}+\mathbf{m}_{\perp} e^{i \omega t}$ with $m_{z} \sim 1$ and $\mathbf{m}_{\perp}=m_{x} \hat{\mathbf{x}}+m_{y} \hat{\mathbf{y}}$, the corresponding dipolar field: $\mathbf{h}=$ $h_{z} \hat{\mathbf{z}}+\mathbf{h}_{\perp} e^{i \omega t}$, and the LLG equation can be linearized in terms of $\mathbf{m}_{\perp}$ and $\mathbf{h}_{\perp}$ :

$$
\mathbf{m}_{\perp}(\mathbf{r})=\chi(\omega) \gamma \mathbf{h}_{\perp}(\mathbf{r}) \quad \text { with } \quad \chi(\omega)=\frac{1}{\hat{\Omega}^{2}-\omega^{2}}\left(\begin{array}{cc}
\hat{\Omega} & i \omega \\
-i \omega & \hat{\Omega}
\end{array}\right)
$$

with $\hat{\Omega}=\omega_{0}+i \alpha \omega-A_{\mathrm{ex}} \nabla^{2}$ and $\omega_{0}=\gamma H_{0}$.

Eq. (7a) implies that the dipolar magnetic field can be expressed in terms of a scalar potential: $\mathbf{h}(\mathbf{r}, t)=-\nabla \psi(\mathbf{r}, t) . \mathbf{b}=\mathbf{h}+\mu_{0} M_{s} \mathbf{h}$ and $\mathbf{m}_{\perp}=\chi \gamma \mathbf{h}_{\perp}$ can also be written in terms of $\mathbf{h}$ and thus $\psi$. Using scalar potential $\psi$, Eq. $(7 \mathrm{~b})$ can be written as

$$
\begin{aligned}
\left(\hat{\Omega}^{2}-\omega^{2}+\omega_{M} \hat{\Omega}\right) \nabla^{2} \psi-\omega_{M} \hat{\Omega} \partial_{z}^{2} \psi=0 & \text { for } \quad-d \leq x \leq 0, \\
\nabla^{2} \psi=0 & \text { for } \quad x<-d \text { or } \quad x>0 .
\end{aligned}
$$

Because of the translational symmetry in the lateral direction, we may assume that the scalar potential is a plane wave of the form:

$$
\psi(x, y, z, t)= \begin{cases}\phi(0) e^{-q x} e^{-i \mathbf{q} \cdot \mathbf{s}} e^{i \omega t} & \text { for } \quad x>0 \\ \phi(x) e^{-i \mathbf{q} \cdot \mathbf{s}} e^{i \omega t} & \text { for } \quad-d \leq x \leq 0 \\ \phi(-d) e^{q(x+d)} e^{-i \mathbf{q} \cdot \mathbf{s}} e^{i \omega t} & \text { for } x<-d\end{cases}
$$

where $\mathbf{s}=(y, z)$ is the in-plane position and $\mathbf{q}=\left(q_{y}, q_{z}\right)=q(\sin \theta, \cos \theta)$ with $q=|\mathbf{q}|$ an inplane wave vector. Using $\psi$ in Eq. (11), Eq. (10b) is valid automatically, and the boundary conditions in Eq. (4) and Eq. (8) become

$$
\begin{array}{r}
\left(1+\frac{\omega_{M} \hat{\Omega}}{\hat{\Omega}^{2}-\omega^{2}}\right) \partial_{x} \psi(x)+\frac{i \omega_{M} \omega}{\hat{\Omega}^{2}-\omega^{2}} \partial_{y} \psi(x) \pm q \psi(x)=0 \quad \text { for } \quad x=0,-d, \\
{\left[\partial_{x}+i k_{p} \frac{\omega}{\omega_{0}}-\left(\begin{array}{cc}
k_{s} & k_{j} \\
-k_{j} & 0
\end{array}\right)\right]\left[\frac{1}{\hat{\Omega}^{2}-\omega^{2}}\left(\begin{array}{cc}
\hat{\Omega} & i \omega \\
-i \omega & \hat{\Omega}
\end{array}\right)\left(\begin{array}{c}
\partial_{x} \\
\partial_{y}
\end{array}\right) \psi(x)\right]=0 \quad \text { for } \quad x=0,} \\
\partial_{x}\left[\frac{1}{\hat{\Omega}^{2}-\omega^{2}}\left(\begin{array}{cc}
\hat{\Omega} & i \omega \\
-i \omega & \hat{\Omega}
\end{array}\right)\left(\begin{array}{c}
\partial_{x} \\
\partial_{y}
\end{array}\right) \psi(x)\right]=0 \quad \text { for } \quad x=-d,
\end{array}
$$


with $\omega_{M}=\gamma \mu_{0} M_{s}$. With Eq. (12), the whole problem is reduced to finding a solution for $\psi$, from which $\mathbf{m}$ and $\mathbf{h}$ can be calculated. To this end we have to solve the differential equations in Eq. (10) subject to the boundary conditions in Eq. (12) [13, 14].

With Eq. (11), Eq. 10a) becomes a 6th-order ordinary differential equation of $\phi(x)$ :

$$
\left[\left(\hat{\Omega}^{2}-\omega^{2}+\omega_{M} \hat{\Omega}\right)\left(\partial_{x}^{2}-q^{2}\right)+\omega_{M} \hat{\Omega} q_{z}^{2}\right] \phi(x)=0 \quad \text { with } \quad \hat{\Omega}=\omega_{0}+i \alpha \omega-A_{\mathrm{ex}}\left(\partial_{x}^{2}-q^{2}\right)
$$

which has the general solution

$$
\phi(x)=\sum_{j=1}^{3}\left[a_{j} e^{i q_{x}^{(j)} x}+b_{j} e^{-i q_{x}^{(j)}(x+d)}\right],
$$

where $q_{x}^{(j)}$ is the $j$-th solution of

$$
\left(\Omega^{2}-\omega^{2}+\omega_{M} \omega\right)\left(q_{x}^{2}+q^{2}\right)-\omega_{M} \Omega q_{z}^{2}=0 \quad \text { with } \quad \Omega=\omega_{0}+i \alpha \omega+A_{\mathrm{ex}}\left(q_{x}^{2}+q^{2}\right) .
$$

and $a_{j}, b_{j}$ are six coefficients to be determined by the six boundary conditions in Eq. (12). By Eqs. (11, 14), the six boundary conditions Eq. (12) can be transformed into a set of linear equations with six unknowns $a_{j}, b_{j}$ :

$$
M(\mathbf{q}, \omega)\left(\begin{array}{c}
a_{j} \\
b_{j}
\end{array}\right)=0
$$

where $M(\mathbf{q}, \omega)$ is a $6 \times 6$ matrix depending on the material parameters and injected spin current: $\omega_{0}, \alpha, k_{s}, k_{j}$. The dipolar-exchange spin wave dispersion is determined by the condition that the determinant of the coefficient matrix vanishes: $|M(\mathbf{q}, \omega)|=0 \Rightarrow \omega(\mathbf{q})$, whose real part represents the energy and imaginary part the inverse lifetime of the spin wave mode. The corresponding solution of Eq. (16) for $a_{j}, b_{j}$ gives the spin wave amplitude profile according to Eq. (11).

The spin wave dispersion in magnetic thin films has been studied long ago [13, 14, 15, but without current-induced spin-transfer torque [16], spin pumping, and/or surface anisotropy. Similar to the Gilbert damping, both the current-induced spin-transfer torque and spin pumping mainly affect the dissipative behavior via the imaginary part of the frequency, while the real part remains practically unchanged. While the surface anisotropy affects the spin wave dispersion itself or the real part of the frequency. From the definition of $\psi$ in Eq. (11), we see that the spin wave is amplified when $\operatorname{Im}[\omega(\mathbf{q})]<0$, which indicates instability and $\operatorname{Im}[\omega(\mathbf{q})]=0$ will be used as criteria for spin wave excitation with wave vector $\mathbf{q}$.

\section{Dispersion, amplification, and dissipation of spin waves in magnetic insulators}

We study in this section the dipole-exchange spin wave dispersion in a thin film of finite thickness. The full spin wave dispersion is anisotropic in $\mathbf{q}$, multiple volume transverse modes exist due to the confinement in the $x$-direction, and magnetostatic surface modes due to the 


\begin{tabular}{c|l|l|c|l|l}
\hline Parameter & YIG & Unit & Parameter & YIG & Unit \\
\hline$M_{s}$ & ${ }^{a} 1.56 \times 10^{5}$ & $\mathrm{~A} / \mathrm{m}$ & $\omega_{0}=\gamma H_{0}$ & $0.5 \omega_{M}$ & $\mathrm{GHz}$ \\
$\alpha$ & ${ }^{a} 6.7 \times 10^{-5}$ & - & $\omega_{M}=\gamma \mu_{0} M_{s}$ & 34.5 & $\mathrm{GHz}$ \\
$g_{r}$ & ${ }^{b} 3 \times 10^{17}$ & $1 / \mathrm{m}^{2}$ & $A_{\text {ex }}$ & $4.74 \times 10^{-6}$ & $\mathrm{~m}^{2} / \mathrm{s}$ \\
$K_{s}$ & ${ }^{c} 5 \times 10^{-5}$ & $\mathrm{~J} / \mathrm{m}^{2}$ & $k_{s}=2 \gamma K_{s} / A_{\mathrm{ex}} M_{s}$ & $2.5 \times 10^{7}$ & $1 / \mathrm{m}$ \\
\hline
\end{tabular}

Table 1: Parameters for YIG. ${ }^{a}$ Ref. [7], ${ }^{b} g_{r}=10^{16} \sim 10^{19} / \mathrm{m}^{2}$, Ref. [7, 17, 18, ${ }^{c} K_{s}=0.01 \sim 0.1 \mathrm{erg} / \mathrm{cm}^{2}$ or $10^{-5} \sim 10^{-4} \mathrm{~J} / \mathrm{m}^{2}$, Ref. [19, 20].

dipolar interactions exist. A proper description contains the following ingredients: finite thickness $(d=0.61 \mu \mathrm{m})$, intrinsic magnetic damping, exchange coupling, and dipolar fields in the bulk, and surface anisotropy, spin current injection, spin pumping at the interface. We calculate numerically the complex eigenfrequencies $\omega\left(\mathbf{q}, k_{j}\right)$ as a function of the in-plane wave vector $\mathbf{q}$ and the injected spin current $k_{j}$. $\operatorname{Im} \omega$, the effective dissipation, can be either positive (damping) or negative (amplification) when driven by the spin-transfer torque. In order to understand the consequences of surface anisotropy, we study three different cases: i) free surface without surface anisotropy, ii) easy-axis surface anisotropy (EASA), and iii) hard-axis (easy-plane) surface anisotropy (HASA). We consider spin pumping in Section 3.5 and 3.4. but initially assume $k_{p}=0$ (zero spin pumping).

\subsection{Without surface anisotropy}

First, we disregard the surface anisotropy: $k_{s}=K_{s}=0$. The solution of Eq. (16) gives the dispersion and dissipation versus the in-plane wave vector $\mathbf{q}$ as shown in Fig. 2 for different angles $\theta=\angle(\mathbf{m}, \mathbf{q})$ between $\mathbf{q}$ and the equilibrium $\mathbf{m}$ (or $\hat{\mathbf{z}}$ ). Without spin current injection, the dispersion (top row) and dissipation (middle row) are the same for $\theta$ and $-\theta$ because the structure is axially symmetric around the $\hat{\mathbf{z}}$-axis. However, the spin current injected on the top surface breaks this symmetry, and the dissipation curves (bottom row) differ for $\theta$ and $-\theta$, while the dispersion remains more or less symmetric because the spin current injection affects the dissipation to first order and dispersion to second order in $k_{j}$.

Let us focus on the results for $\theta=\pi / 2$ in Fig. 3. The left and middle panels show the dispersion Re $\omega$ and dissipation Im $\omega$ without spin current injection. In the plot of Re $\omega$, the magnetostatic surface wave (MSW) crosses the bulk modes [13. The dispersion for the $n$th bulk mode and the (independent) MSW for $\theta=\pi / 2$ and $\alpha \ll 1$ is given by

$$
\begin{aligned}
& \omega_{n}=\sqrt{\omega_{n q}\left(\omega_{n q}+\omega_{M}\right)}+i\left[\left(\alpha+2 \frac{A_{e x} k_{p}}{\omega_{0} d}\right)\left(\omega_{n q}+\frac{\omega_{M}}{2}\right)+2 A_{\mathrm{ex}} \frac{k_{j}}{d}\right] \quad \text { with } n=1,2, \ldots \\
& \omega_{\mathrm{MSW}}=\sqrt{\left(\omega_{0}+\frac{\omega_{M}}{2}\right)^{2}-\frac{\omega_{M}^{2}}{4} e^{-2 q d}}+i\left[\left(\alpha+\frac{A_{e x} k_{p}}{\omega_{0} d}\right)\left(\omega_{0}+\frac{\omega_{M}}{2}\right)+A_{\mathrm{ex}} \frac{k_{j}}{d}\right] \quad \text { for } \quad q d<1 \text {. }
\end{aligned}
$$

with $\omega_{n q}=\omega_{0}+A_{\mathrm{ex}}\left[q^{2}+(n \pi / d)^{2}\right]$. 

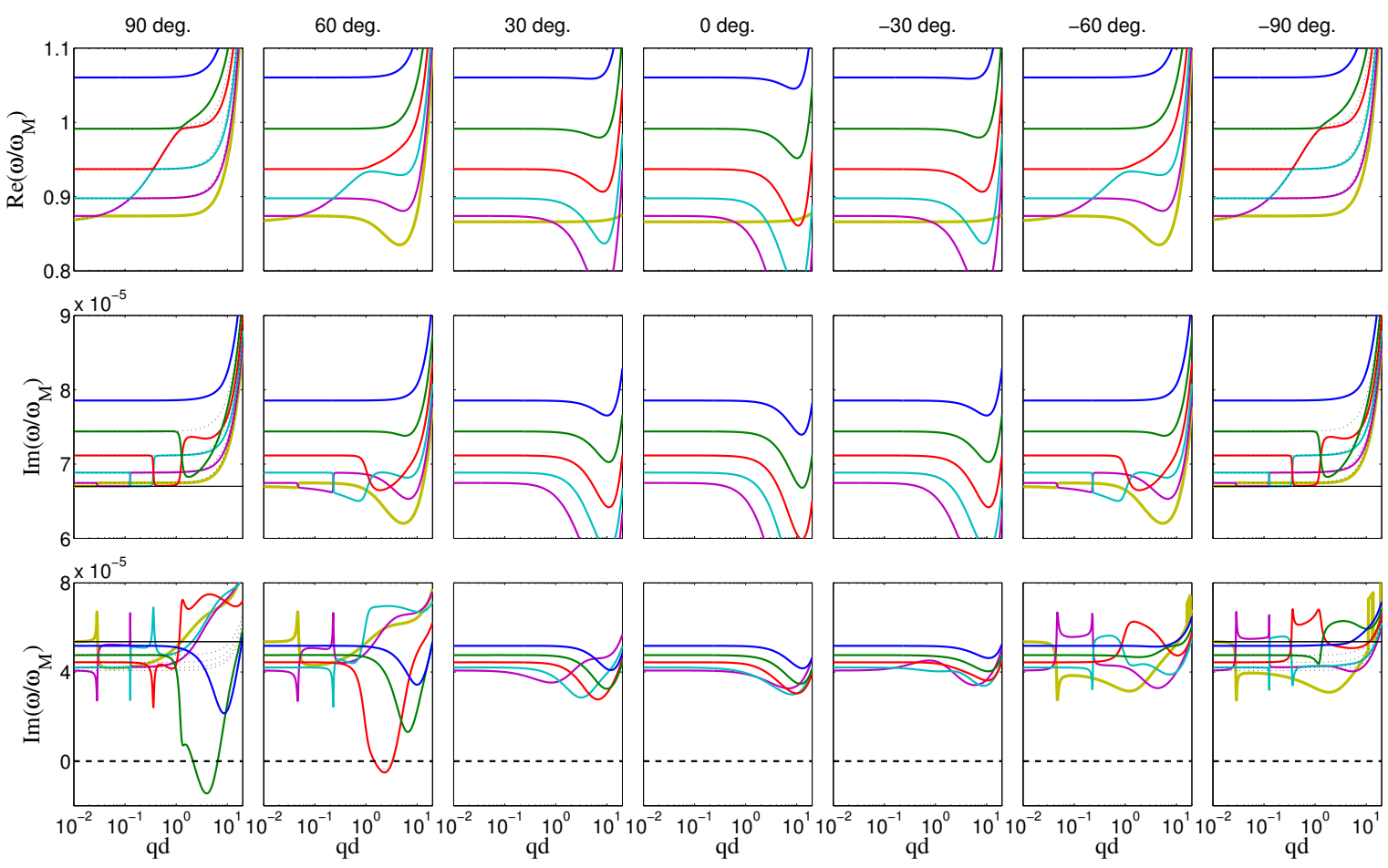

Figure 2: Spin wave dispersion and dissipation at various angle $\theta$ for $k_{s}=0$. Top: Re $\omega$, middle: Im $\omega$ at $k_{j}=0$, bottom: $\operatorname{Im} \omega$ at $k_{j}=-0.2 k_{c}$.

When spin current is injected, Im $\omega$ decreases/increases depending on the polarity of the injected spin current, as expected. For negative $k_{j}$, dissipation decreases for all modes as seen in the right panel of Fig. 3. In the regime $q d<1$, the bulk modes become less damped than the MSW mode as indicated in Eq. (17), which is counter intuitive but can be explained qualitatively using the spin wave profiles in Fig. 4. Here the spin wave amplitude profiles are plotted for the modes indicated by the vertical dashed line in Fig. 3 at two values $q d=0.09,3.74$. Similar to Re $\omega$ the amplitudes are only weakly affected by the spin current injection. The top row in Fig. 4 is for small $q d=0.09 \ll 1$, thus Eq. $17 \mathrm{~b}$ is valid in this regime. The purple (2nd) and the cyan (3rd) curves in the top panels in Fig. 4 show the MSW and the second bulk mode respectively. Though the purple MSW is called a "surface" wave, it is not really localized at the surface because of its small $q: 1 / q \sim d / 0.09 \gg d$, therefore it is close to a uniform mode. The cyan bulk mode is actually "lighter" than the purple MSW mode in terms of total magnetization because of its oscillating magnetization. The dissipation decrease/increase due to the spin current injection is proportional to the boundary value of $\mathbf{m}_{\perp}(0)$ and inversely proportional to the total magnetization of the mode; therefore Im $\omega$ of the bulk mode decreases/increases more strongly here than the MSW mode under spin current injection.

The current-induced dissipation in Eq. (17) is no longer valid for the modes in the bottom 

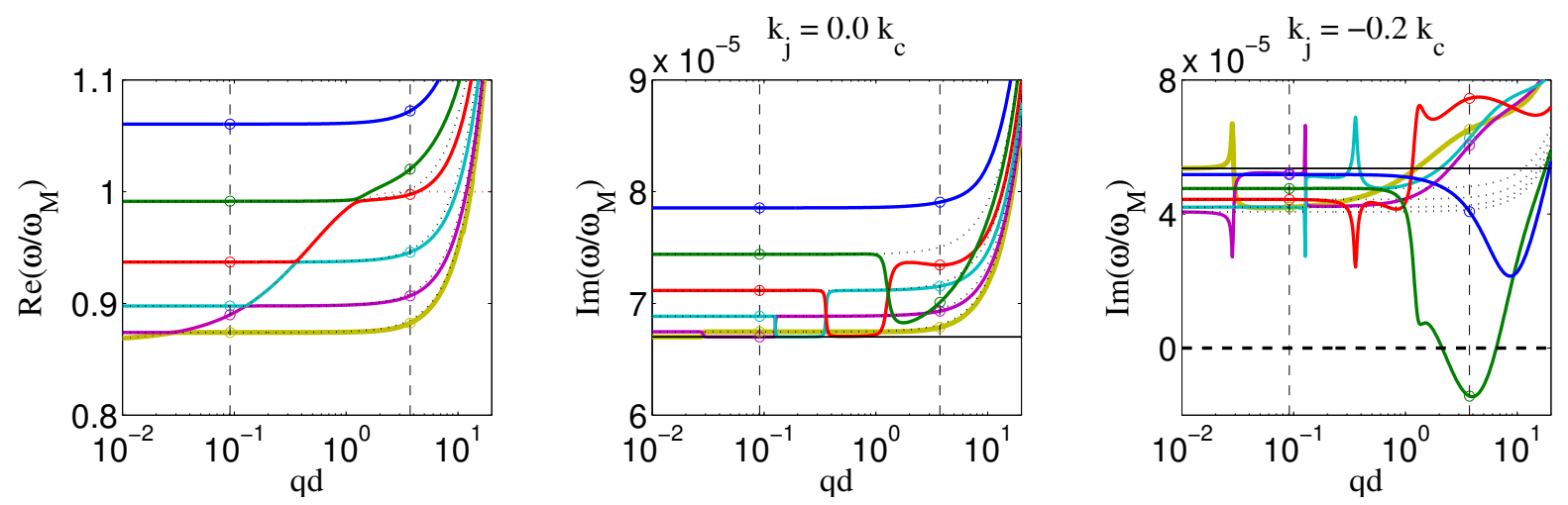

Figure 3: Spin wave band structure in YIG for $d=0.61 \mu \mathrm{m}$ without surface anisotropy $\left(k_{s}=0\right)$ at $\theta=\angle(\mathbf{m}, \mathbf{q})=\pi / 2$. From left to right: $\operatorname{Re} \omega / \omega_{M}, \operatorname{Im} \omega / \omega_{M}$ at $k_{j}=0, \operatorname{Im} \omega / \omega_{M}$ at $k_{j}=-0.2 k_{c}$. The dashed curves are plotted using the analytical expression for $\omega$ in Eq. (17).

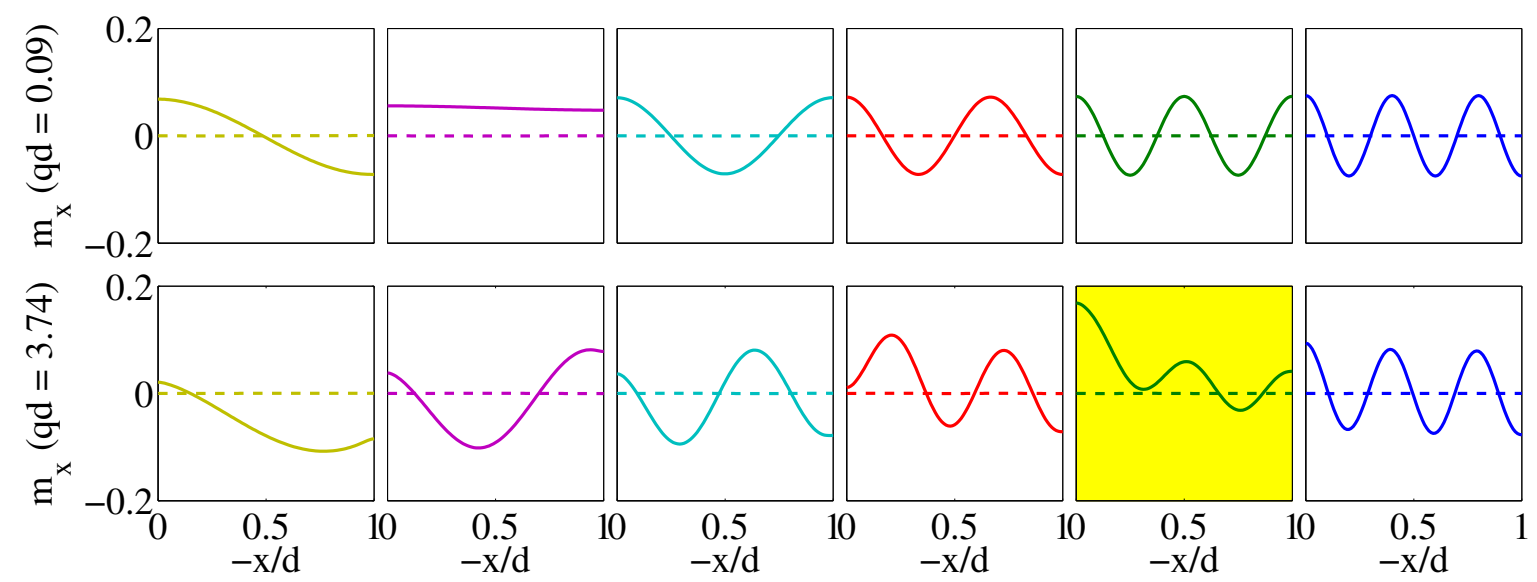

Figure 4: $m_{x}$ of the same 6 modes for $q d=0.09$ and 3.74 indicated by the dashed vertical lines in Fig. 3 The colors label different bands. The amplitude of the green mode mode (shaded/yellow panel) is amplified.

panels in Fig. 4 at $q d=3.74>1$, at which the MSW and the bulk mode hybridize as seen in the red (4th) and green (5th) amplitude profile in the lower panels. Both modes have surface and bulk features. From their profiles, we can infer that the dissipation for the red mode should be weakly influenced by the spin current injection because of its nearly vanishing $\mathbf{m}_{\perp}(0)$, whereas the green mode should be strongly affected because of its large $\mathbf{m}_{\perp}(0)$. Indeed, in the right panel in Fig. 3, the dissipative part $\operatorname{Im} \omega$ for the green mode dips into the negative regime, i.e. is amplified, while the other modes are still damped.

The spiky features in the right-most panel of Fig. 3 are not artifacts: they are caused by mode anti-crossings, at which the two modes form bonding and anti-bonding states. The spin wave profiles $\mathbf{m}(x)$ for the two modes add up (subtract) to form the (anti-)bonding at the anti-crossing point, therefore $\mathbf{m}_{\perp}(0)$ for the two modes also add up (subtract). As the mode transforms from bulk mode to MSW or vice versa when being tuned through the (anti-)crossing, $\mathbf{m}_{\perp}(0)$ decreases/increases first then increases/decreases again. Precisely at 
the anti-crossing point one mode has a maximized $\mathbf{m}_{\perp}(0)$ while that of the other mode is minimal. For example, at the point where the red mode has a dip and the cyan mode has a peak in Fig. 3, $\mathbf{m}_{\perp}$ (0) for the red mode is large while $\mathbf{m}_{\perp}(0)$ for the cyan mode is negligibly small. Therefore, upon the application of spin current, the dissipation for the red mode decreases and the cyan mode is not affected (and is very close to its value for $k_{j}=0$ ).

By its different chirality, an analogous hybrid MSW at $\theta=-\pi / 2$ is localized at the opposite surface to vacuum $(x=-d)$, so $\mathbf{m}_{\perp}(0)$ is small. This means the spin current induced torque at $x=0$ is small and its dissipation is only weakly affected by the spin current injection.

\subsection{With easy-axis surface anisotropy}

We now include the effects of an easy-axis surface anisotropy (EASA) at the top surface. For $k_{s}>0$ the anisotropy energy is minimized when the magnetization is parallel to the surface normal. We assume that the anisotropy is not strong enough to enforce a perpendicular equilibrium magnetization. The solution of Eq. (16) gives the dispersion and dissipation versus the in-plane wave-vector $\mathbf{q}$ which for these conditions are given in Fig. 5 for different angles $\theta$. Different from the $k_{s}=0$ case in Fig. 2, without spin current injection, the dispersion (top row) and dissipation (middle row) are no longer the same for $\theta$ and $-\theta$, because the surface anisotropy is on the top surface only and breaks the axial symmetry around the $\hat{\mathbf{z}}$-axis. Comparing dispersion for $\theta=\pi / 2$ and $\theta=-\pi / 2$ in Fig. 5 and Fig. 2 , we find that the $\theta=\pi / 2$ configuration is modified far more than the $\theta=-\pi / 2$ one by the surface anisotropy. At $\theta=\pi / 2$ case, both MSW and the EASA induce surface spin waves (discussed below) that are localized at the top surface. They are therefore strongly coupled. However, for the $\theta=-\pi / 2$ case, the MSW is localized at the bottom surface, far away from the EASA induced surface spin wave. The spatial separation obviously reduces the coupling and the individual bands remains more or less intact.

Let us focus now on $\theta=\pi / 2$ in order to study the effect of surface anisotropy $\left(k_{s}>0\right.$ for EASA) on the dispersion and dissipation of the spin waves. In the absence of spin current injection $\left(k_{j}=0\right)$, the surface anisotropy shifts the eigenfrequency of the $n$-th bulk mode to (assuming $A_{\mathrm{ex}} k_{s}^{2} \ll \omega_{0}$ and $q d \lesssim 1$ ):

$$
\omega_{n}^{\prime}=\omega_{n}-\frac{A_{\mathrm{ex}} k_{s}}{d}\left[\frac{\omega_{n q}}{\operatorname{Re} \omega_{n}}\left(1-\frac{\omega_{n q}+\omega_{M}}{2 \omega_{n q}+\omega_{M}} \sqrt{\frac{A_{\mathrm{ex}} k_{s}^{2}}{\omega_{n q}+\omega_{0}+\omega_{M}}}\right)^{-1}+i \alpha\right],
$$

where $\omega_{n}$ is given by Eq. (17a) for $k_{s}=0$. Both frequency and dissipation decrease with increasing surface anisotropy $k_{s}$. We may compare Eq. (18) (thin dashed line) with the numerical calculations (thick solid line) in Fig. 6. At zero spin current injection (the left and middle panels), both real and imaginary parts are well represented by Eq. (18) in the present parameter regime.

Apart from the eigenfrequency shifts of the bulk modes, the main differences between $k_{s}=0$ (Fig. 3) and $k_{s}>0$ (Fig. 6) cases are: i) appearance of an additional (black) band, 


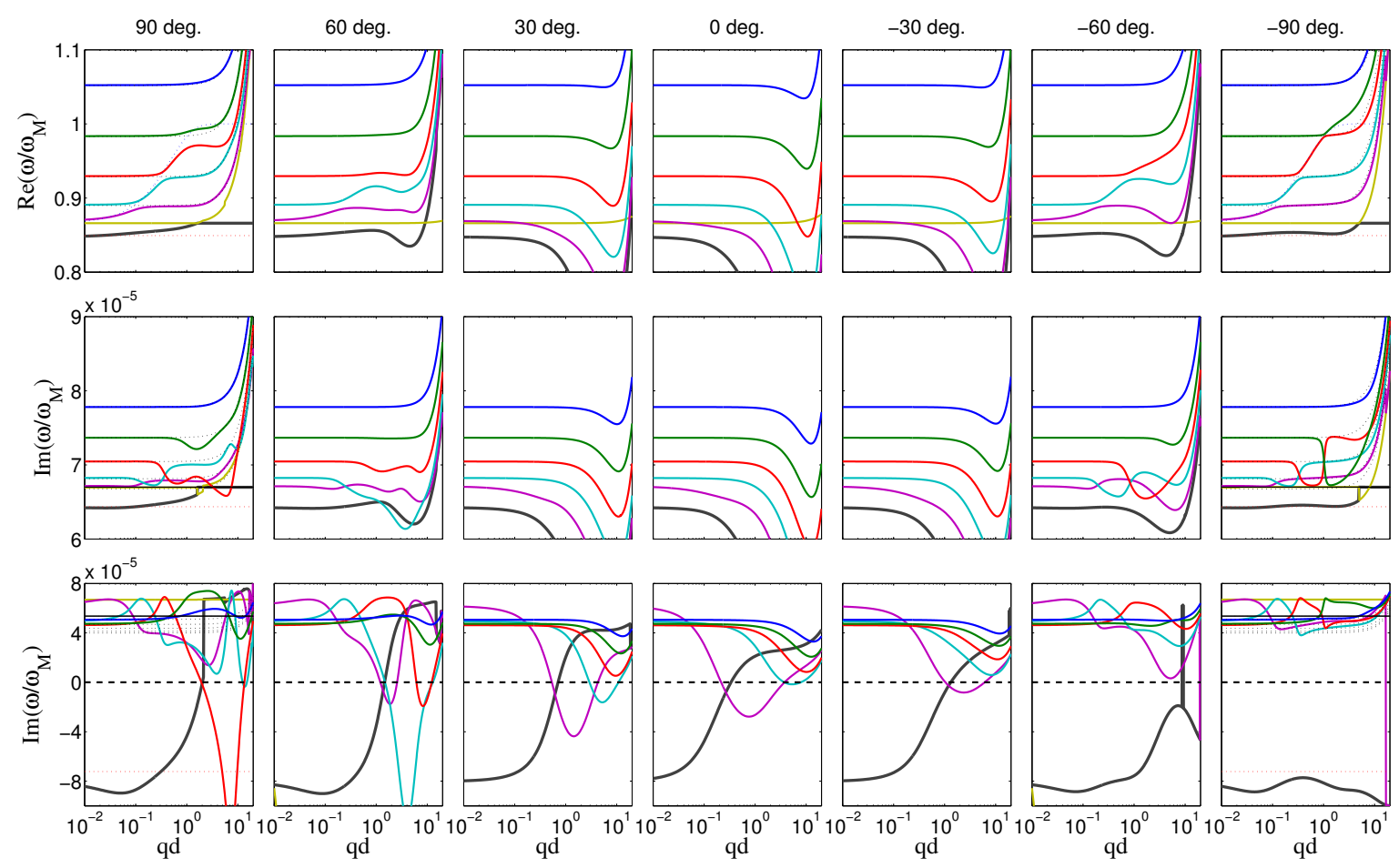

Figure 5: Spin wave dispersion and dissipation at various angle $\theta$ for $k_{s}=25 / \mu \mathrm{m}$. Top: Re $\omega$, middle: $\operatorname{Im} \omega$ at $k_{j}=0$, bottom: $\operatorname{Im} \omega$ at $k_{j}=-0.2 k_{c}$.

whose frequency (at $q=0$ ) is less than the lowest macrospin mode frequency $\sqrt{\omega_{0}\left(\omega_{0}+\omega_{M}\right)}$, ii) strongly modified spin wave hybridization for $q d \gtrsim 1$ by the new band in i).

In order to understand the differences caused by EASA, we study the limit $d \rightarrow \infty$, i.e. the magnetic film is semi-infinite and $b_{j}=0$ in Eq. (14). Focusing for simplicity on vanishing in-plane wave-vector $\mathbf{q}=\left(q^{y}, q^{z}\right)=0$, Eq. (13) becomes of 4th-order and the scalar potential can be written as:

$$
\psi(\mathbf{r})=\sum_{j=1}^{2} a_{j} e^{i q_{j} x} e^{i \omega t} \quad \text { with } \quad q_{j}(\omega)=-i\left(\frac{\omega_{0}+\frac{1}{2} \omega_{M} \pm \sqrt{\omega^{2}+\frac{1}{4} \omega_{M}^{2}} \pm i \alpha \omega}{A_{\mathrm{ex}}}\right)^{\frac{1}{2}}
$$

where $q_{1,2}$ are negatively imaginary with $\left|q_{1}\right| \gg\left|q_{2}\right|$. Imposing the boundary conditions from Eq. (12) at $x=0,|M(\mathbf{q}, \omega)|=0$ leads to (up to the first order in $k_{j}$ ):

$$
2 q_{1} q_{2}\left(q_{1}+q_{2}\right)+i k_{s}\left[\left(q_{1}+q_{2}\right)^{2}+\frac{\omega_{M}}{A_{\mathrm{ex}}}\right]+4 k_{j} \omega=0 .
$$

The solutions of Eq. 20 are the complex eigenfrequencies $\omega$.

To 0th-order in dissipation, i.e. with vanishing bulk damping $(\alpha=0)$ and spin current injection $\left(k_{j}=0\right)$, Eq. (20) simplifies to $k_{s}=2 i q_{2} /\left[1+\omega_{M} /\left(A_{\mathrm{ex}} q_{1}^{2}\right)\right]$ using $\left|q_{1}\right| \gg\left|q_{2}\right|$. 

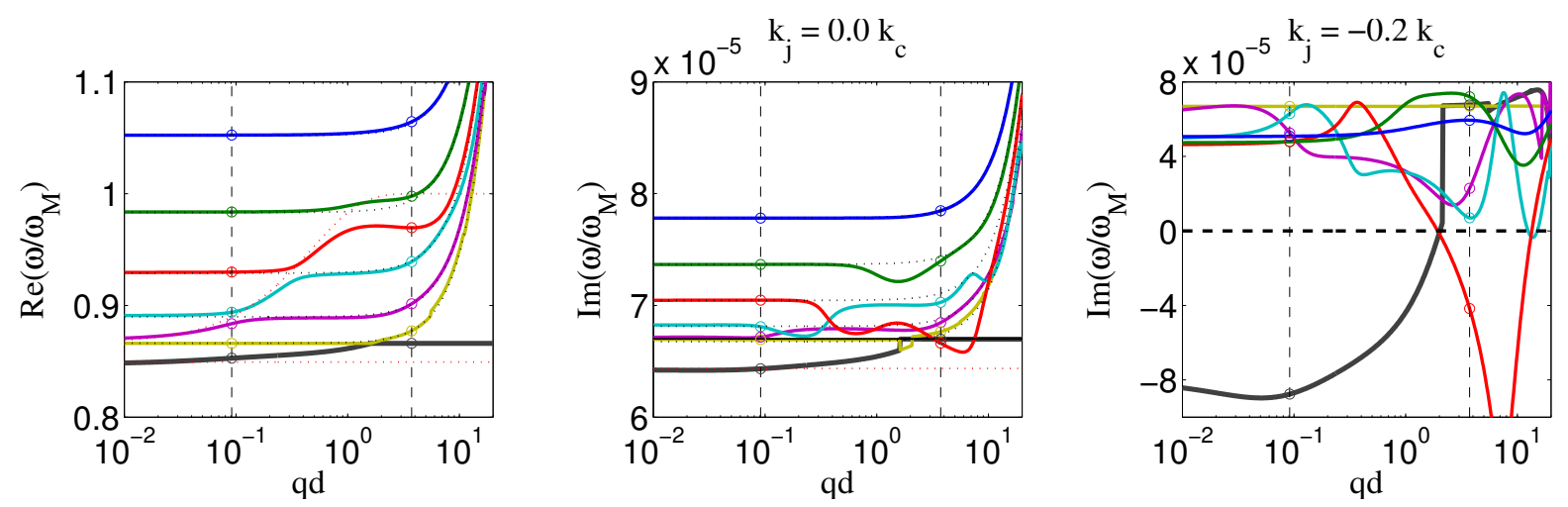

Figure 6: Same as Fig. 3 but with $k_{s}=25 / \mu \mathrm{m}$. The black (red) dashed curves are plotted using the analytical expression in Eq. (18) (Eqs. 21, 22) for the bulk modes (EASA surface wave) at zero spin current.

There is no real solution when surface normal is the hard axis or $k_{s} \leq 0$. However, when the surface normal is an easy axis or $k_{s}>0$, there is a single real solution $\omega_{S}$. For $\eta_{s} \equiv \sqrt{A_{\mathrm{ex}} k_{s}^{2} /\left(2 \omega_{0}+\omega_{M}\right)} \ll 1$

$$
\begin{aligned}
\operatorname{Re} \omega_{\mathrm{S}} & \simeq \sqrt{\omega_{0}\left(\omega_{0}+\omega_{M}\right)}\left[1-\eta_{s} \frac{\omega_{0}}{2\left(\omega_{0}+\omega_{M}\right)}-\eta_{s}^{2} \frac{\omega_{0}}{2 \omega_{0}+\omega_{M}}\right] \\
q_{1} & \simeq-i \sqrt{\frac{2 \omega_{0}+\omega_{M}}{A_{\mathrm{ex}}}} \text { and } q_{2} \simeq-i \sqrt{\frac{\omega_{0}^{2} /\left(2 \omega_{0}+\omega_{M}\right)}{A_{\mathrm{ex}}}}\left(\eta_{s}+\eta_{s}^{2} \frac{\omega_{0}+\omega_{M}}{2 \omega_{0}+\omega_{M}}\right) .
\end{aligned}
$$

Since $q_{1,2}$ are both negative imaginary, the corresponding spin waves in Eq. (19) are localized near the surface. Because $\left|q_{1}\right| \gg\left|q_{2}\right|$, the penetration depth of a surface wave is roughly $d_{s}=1 /\left|q_{2}\right| \propto 1 / k_{s}$, which proves the direct involvement of the surface anisotropy. Note that, in contrast to the MSW, the EASA-induced surface spin wave is not chiral, i.e. its amplitude profiles are identical for $\mathbf{q}$ and $\mathbf{- q}$ (if not hybridized with the MSW).

To the 1st-order in the dissipation, i.e. in $\alpha$ and $k_{j}$, we find

$$
\begin{aligned}
\operatorname{Im} \omega_{\mathrm{S}} & =i \sqrt{\operatorname{Re} \omega_{\mathrm{S}}^{2}+\frac{\omega_{M}^{2}}{4}}\left[1-\eta_{s}^{2} \frac{2 \omega_{0} \omega_{M}}{\left(2 \omega_{0}+\omega_{M}\right)^{2}}\left(1+\eta_{s} \frac{2 \omega_{M}^{2}-\omega_{0}^{2}}{\omega_{M}^{2}+2 \omega_{0} \omega_{M}}\right)\right] \\
& \times\left\{\alpha+\eta_{s} \sqrt{\frac{A_{\mathrm{ex}}}{2 \omega_{0}+\omega_{M}}}\left[k_{j} \frac{2 \omega_{0}}{2 \omega_{0}+\omega_{M}}\left(1+\eta_{s} \frac{\omega_{0}+2 \omega_{M}}{2 \omega_{0}+\omega_{M}}\right)+k_{p}\left(1+\eta_{s} \frac{\omega_{0}+\omega_{M}}{2 \omega_{0}+\omega_{M}}\right)\right]\right\} .
\end{aligned}
$$

The eigenfrequencies in Eqs. (21, 22) are derived for $\mathbf{q}=0$ and for a semi-infinite film. However, we still expect similar surface spin wave for film thickness $d \gg 1 / k_{s}$. For a YIG thin film with $d=0.61 \mu \mathrm{m}$, using the parameters given in Table 1, we estimate the penetration depth $d_{s} \simeq 1 /\left|q_{2}\right|=0.13 \mu \mathrm{m} \ll d$, so Eqs. (21, 22) still hold. The frequency and dissipation given in Eqs. 21, 22) are shown as the red dashed horizontal line in Fig. 6. which agrees very well with the black band in the same figure at the point $q=0$. 


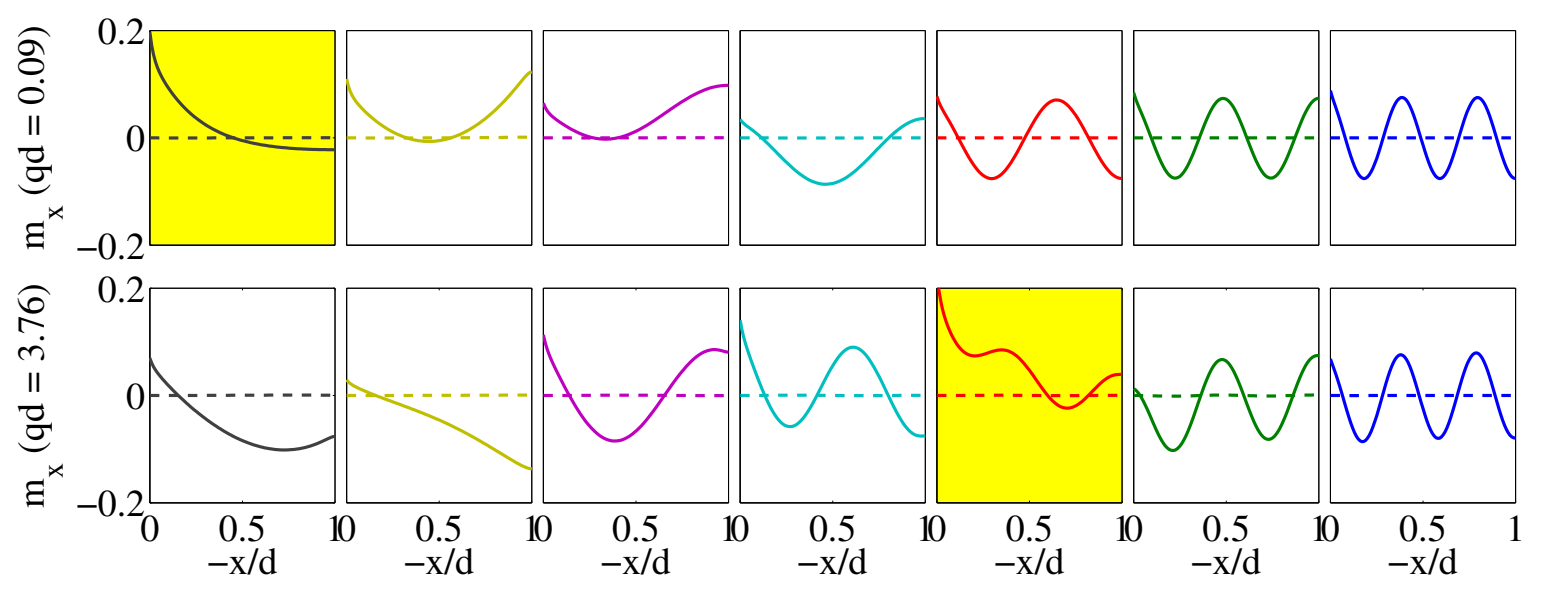

Figure 7: Same as Fig. 4 but with $k_{s}=25 / \mu \mathrm{m}$.

According to Eq. (22) the imaginary part of the eigenfrequency for the surface mode becomes negative when

$$
k_{j}<k_{j}^{c}=-\frac{\alpha}{k_{s}} \frac{\left(2 \omega_{0}+\omega_{M}\right)^{2}}{4 A_{\mathrm{ex}} \omega_{0}}+\alpha \frac{\omega_{0}+2 \omega_{M}}{4 \omega_{0}} \sqrt{\frac{2 \omega_{0}+\omega_{M}}{A_{\mathrm{ex}}}}+\frac{2 \omega_{0}+\omega_{M}}{2 \omega_{0}} k_{p},
$$

where $k_{j}^{c}$ is the threshold current for exciting the EASA induced surface spin wave. When $A_{\text {ex }} k_{s}^{2} \ll \omega_{0}$, the first term in Eq. (23) dominates and the threshold current is proportional to the penetration depth of the surface spin wave, $d_{s} \sim 1 / k_{s}$, because the threshold current is directly proportional to the total magnetization that is excited. Using the parameters in Table 1, the threshold current for exciting the EASA induced surface spin wave $($ at $\mathbf{q}=0)$ is $k_{j}^{c}=-0.08 k_{c}$.

The spin current injection has little effect on the dissipation of MSW because the MSW is only well defined for $q d \lesssim 1$ and its decay length is comparable to or larger than the thickness of the film $d$, which means that a MSW at small wave vectors is similar to the bulk modes. On the other hand, the EASA-induced surface spin wave decays with $d_{s} \sim 1 / k_{s}$ and is strongly localized near the surface for sufficiently large $k_{s}$. Therefore the spin current injection can strongly affect this mode because of its small total magnetization [21]. As seen in the right panel of Fig. 6, almost the whole black band is strongly amplified by a spin current injection of $k_{j}=-0.2 k_{c}$.

Inspecting the spin wave profiles in Fig. 7 at two different $q$ values, we observe a surface spin wave near $x=0$ for the black band at small $q$ (top yellow panel). At larger $q$ (bottom panels), the 1st (black) band loses its surface wave features to the 5th (red) band (see right panel in Fig. 6). The red band mode starts out as a MSW, but the EASA enhances its surface localization by hybridization with the black mode to become strongly amplified by the spin current at higher $q$, which is seen in the lower yellow panel of Fig. 7. 

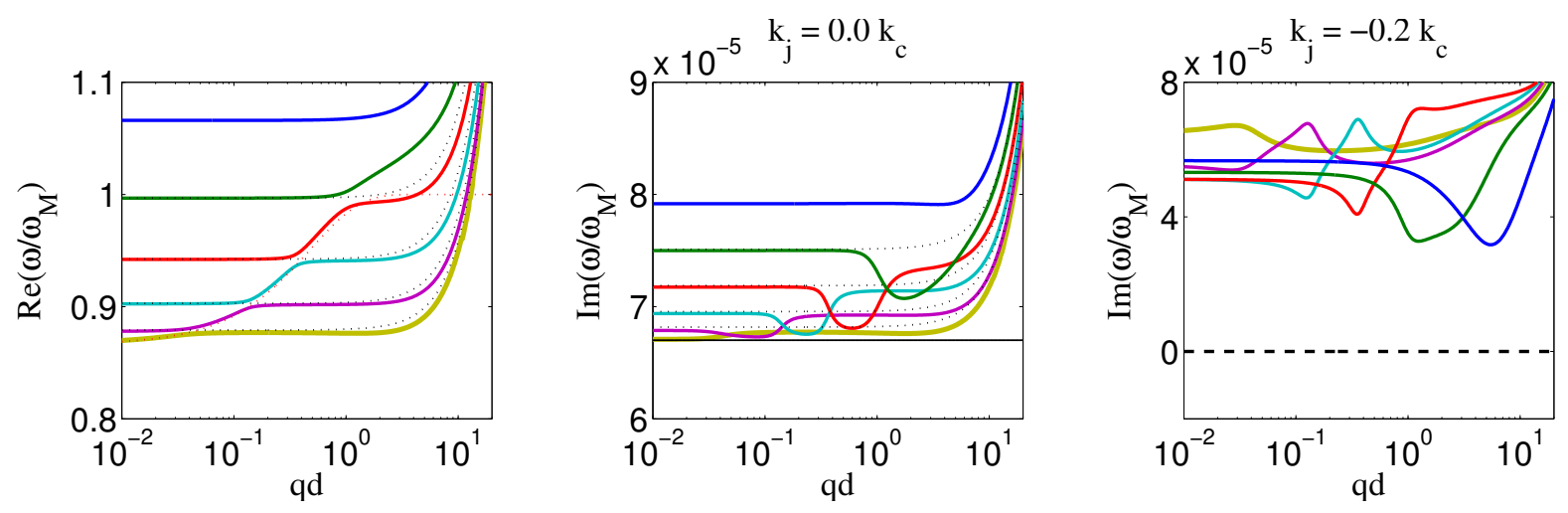

Figure 8: Same as Fig. 3 but with $k_{s}=-25 / \mu \mathrm{m}$. The black dotted curves are plotted using Eq. 18.

\subsection{With hard-axis surface anisotropy}

As mentioned in the previous subsection, there is no surface mode solution to Eq. (20) in the presence of a hard-axis surface anisotropy $\left(k_{s}<0\right)$ favoring interface spins that lie in plane. The dispersion for $k_{s}<0$ shown in Fig. 8 is similar to that for $k_{s}=0$ except for a shift of the bulk modes as expected from Eq. (18). However, we observe that the spin current has little effect now since all modes avoid the interface such that $\mathbf{m}_{\perp}(0)$ is small.

\subsection{Excitation power spectrum}

We introduce an approximate power spectrum that summarizes the information about the mode-dependent current-induced amplification as a sum over bands with band index $n$ :

$$
P(\omega)=\sum_{n} \int_{\operatorname{Im} \omega_{n}<0}\left|\operatorname{Im} \omega_{n}(\mathbf{q})\right| \delta\left[\omega-\operatorname{Re} \omega_{n}(\mathbf{q})\right] \mathrm{d} \mathbf{q},
$$

which is the density of states at frequency $\omega$ weighted by its amplification. As a disclaimer, we note that Eq. (24) merely gives partial information about the excitation ignoring e.g. their spin pumping once excited. It fails to describe any self-organization due to the coupling between excited modes. Nevertheless, it possibly provides a qualitative picture of the power spectrum near the threshold.

Without surface anisotropy (see Fig. 9 (a)), only a few modes are excited even at a relatively large current. However, when $k_{s}=25 / \mu \mathrm{m}$ as shown in Fig. 9 (b), the excitation is strongly enhanced by more than two orders of magnitude due to the easily excitable surface spin wave modes. Furthermore, we observe broadband excitation over a much larger range of frequencies. This power spectrum is rather smooth, while the experiments by Kajiwara et al. [7] show a large number of closely spaced peaks. The latter fine structure is caused by size quantization of spin waves due to the finite lateral extension of the sample that has not been taken into account in our theory since it complicates the calculations without introducing new physics. The envelope of the experimental power spectrum compares favorably with the present model calculations. The insets in Fig. 9 show the integrated power and allow the following conclusions: 1) the excitation power is enhanced by at least two orders of magnitude by the EASA; 2) the critical current for magnetization dynamics is $k_{j} \sim 0.08 k_{c}$ 

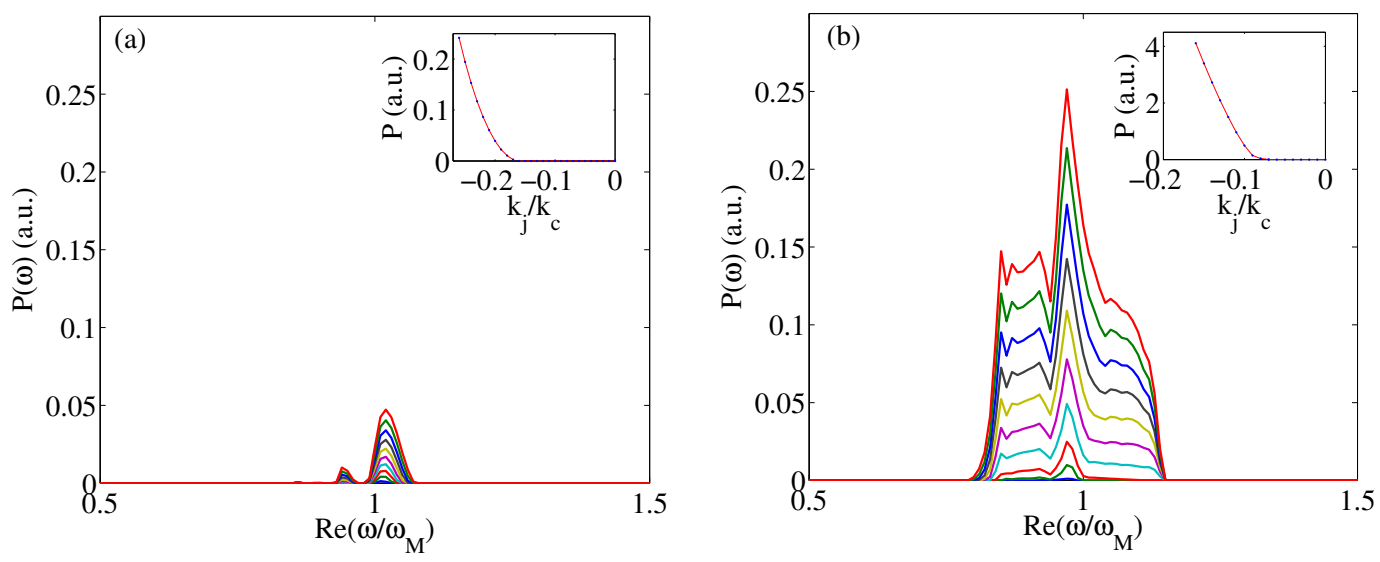

Figure 9: Power spectrum (resolution $\delta \omega / \omega_{M}=0.01$ ) at various current levels (decreasing by $\delta k_{j}=0.01 k_{c}$ ): (a) $k_{s}=0$, (b) $k_{s}=25 / \mu \mathrm{m}$; We plot ten current levels exceeding the threshold critical current. Insets: the integrated power versus $k_{j}$.

for $k_{s}=25 / \mu \mathrm{m}$, which agrees very well with the estimates from Eq. (23). This critical current is about one order of magnitude smaller than that for the bulk excitation $\left(k_{c}\right)$, and about half of that for the case without surface anisotropy $\left(k_{j}=-0.16 k_{c}\right)$. These values are calculated for a film thickness of $d=0.61 \mu \mathrm{m}$, but should not change much for $d=1.3 \mu \mathrm{m}$ corresponding to the experiment [7], because the excited spin waves are localized at the interface.

\subsection{Spin pumping}

The effect of spin pumping depends on the spin-mixing conductance at the interface, which also governs the spin-transfer torque. A complete theory requires self-consistent treatment of both effects on an equal footing, which is still under study. In this section, we briefly discuss the implications of spin pumping. The values quoted for the spin mixing conductance vary strongly, i.e. between $10^{16} \sim 10^{20} / \mathrm{m}^{2}$ [7, 17, 18, 22, 23]. A mixing conductance of $g_{r}=3 \times 10^{17} / \mathrm{m}^{2}$ as listed in Table 1 corresponds to $k_{p}=0.01 / \mu \mathrm{m}$. This value is of the same order of magnitude as the Gilbert damping, i.e. $k_{p} \sim \alpha \omega_{0} d / A_{e x}$ in Eq. (17a). If $g_{r}$ is increased by one order of magnitude as predicted by first-principles calculations [23] and confirmed experimentally [17, 18], the spin pumping will overwhelm the Gilbert damping, but negligible when smaller by a factor of 10. Similar to the Gilbert damping and spin-transfer torque, spin pumping mainly affects the imaginary part of the frequency and modifies the dissipation of the spin wave as in Eqs. (17, 22). Eqs. (17, 22) also indicate that spin pumping enhances magnetic damping, and counteracts the spin-transfer torque for $k_{j}<0$.

In linear response, the effect of spin-transfer torque and spin pumping on the spin wave dissipation are simply additive and therefore plotted separately in Fig. 10. The top two panels are the changes of the dissipation $\delta \operatorname{Im} \omega=\operatorname{Im}\left(\omega_{k_{j}=-0.2 k_{c}}\right)-\operatorname{Im}\left(\omega_{k_{j}=0}\right)$ due to the 

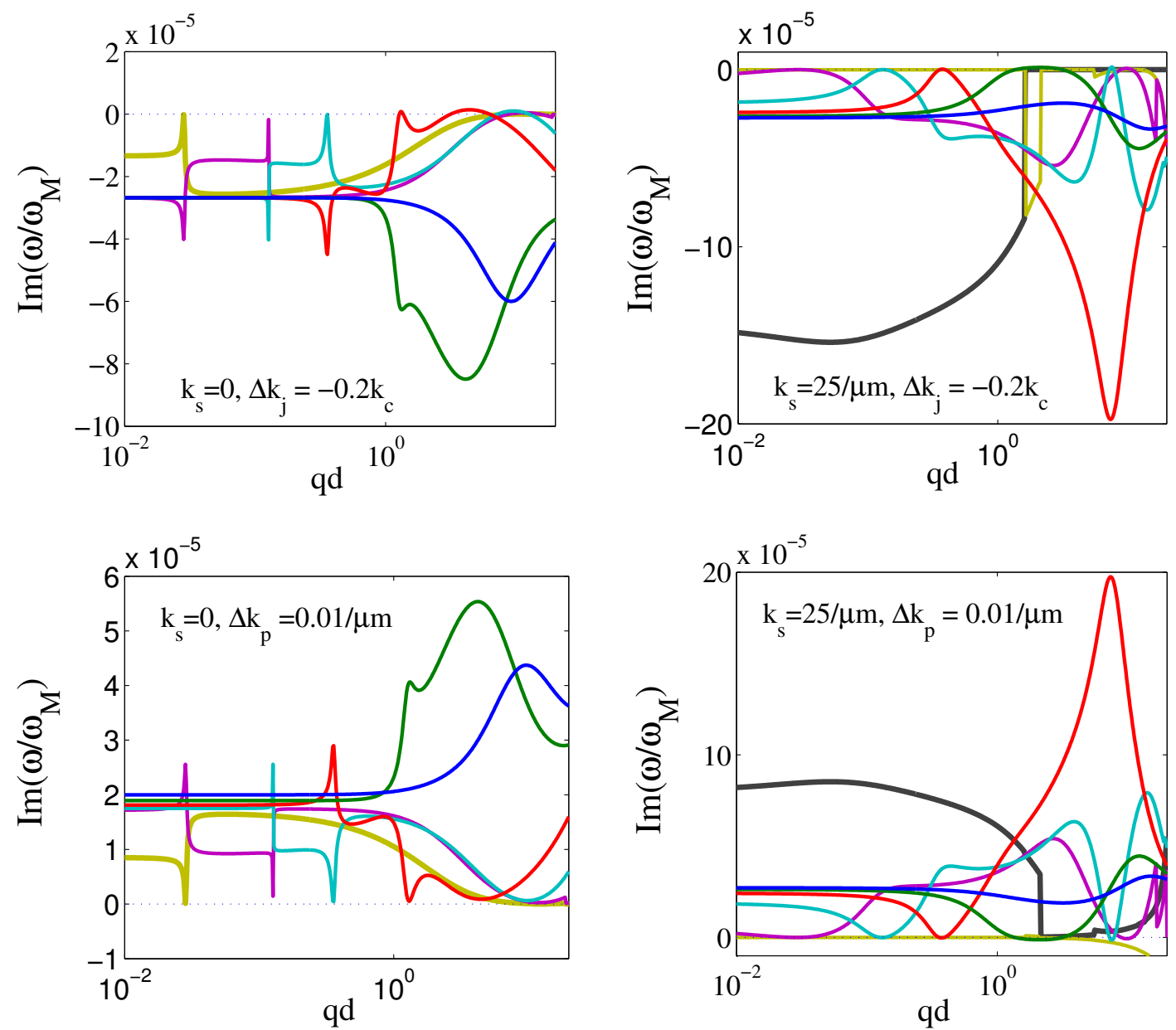

Figure 10: Effects on spin wave dissipation by the spin transfer torque $k_{j}=-0.2 k_{c}$ (the top two panels), obtained by subtracting the results for $k_{j}=0, k_{p}=0$ from that of $k_{j}=-0.2 k_{c}, k_{p}=0$; the band shift due to spin pumping $k_{p}=0.01 / \mu \mathrm{m}$ (the bottom two panels), obtained by subtracting the spin wave bands when $k_{j}=0, k_{p}=0$ from the spin wave bands with spin pumping only $\left(k_{j}=0, k_{p}=0.01 / \mu \mathrm{m}\right)$.

spin-transfer torque $\left(k_{p}=0\right)$, while the bottom two panels are $\delta \operatorname{Im} \omega=\operatorname{Im}\left(\omega_{k_{p}=0.01 / \mu m}\right)-$ Im $\left(\omega_{k_{p}=0}\right)$ represent the effect of spin pumping only $\left(k_{j}=0\right)$. As both are proportional to the boundary value of magnetization, spin-transfer torque and spin pumping (for the chosen value of $k_{p}$ ) induce almost identical changes, but with opposite signs. The relative change for different modes can be understood by the boundary value of magnetization $\mathbf{m}_{\perp}(0)$ observed in the spin wave profiles in Fig. 4 and Fig. 7. Clearly, the MSW and EASA induced surface modes are more strongly affected by the spin pumping [24], thereby partly compensating the reduced critical current reported above for the same modes. 


\section{Discussion}

EASA enables a surface spin wave for the following reason: when $k_{j}=J_{s}=0$, the boundary condition in Eq. (4) requires cancellation of the exchange and surface anisotropy torques: $\partial_{x} m_{x}-k_{s} m_{x}=\partial_{x} m_{y}=0$ at $x=0$. The exchange torque depends on the magnetization derivative in the normal direction, and can only take one sign in the whole film, and $m_{x, y} \rightarrow 0$ as $x \rightarrow-\infty$, therefore $\left(1 / m_{x}\right) \partial_{x} m_{x}>0$. Torque cancellation (for a non-trivial solution) is therefore possible only for $k_{s}>0$, which corresponds to an easy axis anisotropy (EASA). This EASA-induced surface spin wave only exists for the in-plane magnetized film $\left(m_{z} \sim 1\right)$ as discussed here. For comparison, another type of surface spin waves exists for the perpendicular magnetized film $\left(m_{x} \sim 1\right)$ with easy-plane surface anisotropy for $k_{s}<0$, for which the boundary condition is $\partial_{x} m_{y, z}+k_{s} m_{y, z}=0$ at $x=0$ [25, 26, 27, 15, 11].

According to Eq. (23), critical current (excitation power) would be further reduced (increased) by a larger EASA. Ref. [19] reports an enhancement of the YIG surface anisotropies for capped as compared to free surfaces. A Pt cover on a YIG surface [7] may enhance the surface anisotropy as well. As seen from Fig. 6, the surface mode (black band) has group velocity $\partial \omega / \partial \mathbf{q}$ comparable to that of the MSW. The excited surface spin wave therefore propagate and can be used to transmit spin information over long distance at a much lower energy cost than the bulk spin waves. On the other hand, they will also be more easily scattered by surface imperfections.

The surface anisotropies of magnetic thin films with interface to vacuum or other materials are not well known. According to Eq. (18), the bulk mode eigen-frequencies shift under the surface anisotropy. Brillouin light scattering experiments of the spin wave frequencies can therefore provide which gives direct information that can help determining both positive and negative $k_{s}$, unlike the EASA-induced surface wave, which only exists for $k_{s}>0$. In the latter case, according to Eq. (21), it is also possible to determine the value of $k_{s}$ by measuring the eigen-frequency of the EASA-induced surface wave.

The EASA induced surface spin waves have the following properties, i) they can be easily induced (intentionally or unintentionally) by engineering the surface anisotropy, ii) its penetration depth is controllable by the strength of the surface anisotropy, iii) its excitation requires only small spin currents, iv) it has finite group velocity and can propagate long distance without much loss in the absence of surface roughness. The EASA induced surface spin wave is strongly localized at the surface, depending on the strength of EASA. This property means that the EASA surface waves are strongly damped by spin pumping, but only weakly absorb microwaves. Da Silva et al. indeed observed such behavior in a recent experiment [28]. Because the EASA surface wave only exists when there is EASA, it likely does not propagate naturally in uncovered sections of the film without EASA. In such circumstances, some intermode scattering would occur. This property could be used advantageously, e.g. in order to fabricate surface spin wave guides on YIG films. If the complication due to the enhanced spin pumping can be controlled, this type of surface spin wave mode might be superior for spin information processing and transport.

In the present study we were mainly concerned with the magnetization dynamics, disregarding the details of electron and spin transport in the normal metal, such as the spin 
current generation, spin accumulation at the interface [8]. Although the values of the parameters of spin current generation by the inverse spin Hall effect are still under debate, a more quantitative study of the transport processes in the normal metal is called for.

In conclusion, we predict that an easy-axis surface anisotropy gives rises to a surface spin wave mode, which reduces the threshold current required to excite the spin waves and dramatically increases the excitation power. Multiple spin wave modes can be excited simultaneously at different frequencies and wave vectors, thereby explaining recent experiments. Surface spin wave excitations could be useful in low-power future spintronics-magnonics hybrid circuits.

\section{Acknowledgment}

This work was supported by the National Natural Science Foundation of China (No. 11004036, No. 91121002), the special funds for the Major State Basic Research Project of China (No. 2011CB925601), the University Grant Council (AoE/P-04/08) of the government of HKSAR, the FOM foundation, DFG Priority Program SpinCat, EG-STREP MACALO, the ICC-IMR, and ReiMei project.

\section{References}

\section{References}

[1] L. Berger, Emission of spin waves by a magnetic multilayer traversed by a current, Physical Review B $54(13)(1996) 9353$.

[2] J. C. Slonczewski, Current-driven excitation of magnetic multilayers, Journal of Magnetism and Magnetic Materials 159 (1-2) (1996) L1.

[3] A. Brataas, A. D. Kent, H. Ohno, Current-induced torques in magnetic materials, Nature Materials 11 (5) (2012) 372-381.

[4] E. Saitoh, M. Ueda, H. Miyajima, G. Tatara, Conversion of spin current into charge current at room temperature: Inverse spin-hall effect, Applied Physics Letters 88 (18) (2006) 182509-3.

[5] A. A. Serga, A. V. Chumak, B. Hillebrands, YIG magnonics, Journal of Physics D: Applied Physics $43(26)(2010) 264002$.

[6] A. Khitun, D. E. Nikonov, M. Bao, K. Galatsis, K. L. Wang, Feasibility study of logic circuits with a spin wave bus, Nanotechnology 18 (46) (2007) 465202.

[7] Y. Kajiwara, K. Harii, S. Takahashi, J. Ohe, K. Uchida, M. Mizuguchi, H. Umezawa, H. Kawai, K. Ando, K. Takanashi, S. Maekawa, E. Saitoh, Transmission of electrical signals by spin-wave interconversion in a magnetic insulator, Nature 464 (7286) (2010) 262-266.

[8] Y. Tserkovnyak, A. Brataas, G. E. W. Bauer, Enhanced gilbert damping in thin ferromagnetic films, Physical Review Letters 88 (11) (2002) 117601.

[9] M. Madami, S. Bonetti, G. Consolo, S. Tacchi, G. Carlotti, G. Gubbiotti, F. B. Mancoff, M. A. Yar, J. Akerman, Direct observation of a propagating spin wave induced by spin-transfer torque, Nature Nanotechnology 6 (10) (2011) 635-638.

[10] Z. Wang, Y. Sun, M. Wu, V. Tiberkevich, A. Slavin, Control of spin waves in a thin film ferromagnetic insulator through interfacial spin scattering, Physical Review Letters 107 (14) (2011) 146602.

[11] A. G. Gurevich, G. A. Melkov, Magnetization oscillations and waves, CRC Press, P, 1996.

[12] M. D. Stiles, A. Zangwill, Anatomy of spin-transfer torque, Physical Review B 66 (1) (2002) 014407.

[13] R. E. De Wames, Dipole-exchange spin waves in ferromagnetic films, Journal of Applied Physics 41 (3) (1970) 987. 
[14] B. Hillebrands, Spin-wave calculations for multilayered structures, Physical Review B 41 (1) (1990) $530-540$.

[15] B. A. Kalinikos, A. N. Slavin, Theory of dipole-exchange spin wave spectrum for ferromagnetic films with mixed exchange boundary conditions, Journal of Physics C: Solid State Physics 19 (1986) 70137033.

[16] J. Xiao, G. E. W. Bauer, Spin-wave excitation in magnetic insulators by spin-transfer torque, Physical Review Letters 108 (21) (2012) 217204.

[17] C. Burrowes, B. Heinrich, B. Kardasz, E. A. Montoya, E. Girt, Y. Sun, Y.-Y. Song, M. Wu, Enhanced spin pumping at yttrium iron garnet/au interfaces, Applied Physics Letters 100 (9) (2012) 092403. doi:10.1063/1.3690918,

[18] F. D. Czeschka, L. Dreher, M. S. Brandt, M. Weiler, M. Althammer, I.-M. Imort, G. Reiss, A. Thomas, W. Schoch, W. Limmer, H. Huebl, R. Gross, S. T. B. Goennenwein, Scaling behavior of the spin pumping effect in ferromagnet-platinum bilayers, Physical Review Letters 107 (4) (2011) 046601.

[19] P. Yen, T. S. Stakelon, P. E. Wigen, Magnetic-surface interactions in thin yttrium iron garnet films, Physical Review B 19 (9) (1979) 4575.

[20] O. G. Ramer, C. H. Wilts, The effects of surface layers on spin-wave spectra in YIG films, physica status solidi (b) 73 (2) (1976) 443-453.

[21] C. W. Sandweg, Y. Kajiwara, K. Ando, E. Saitoh, B. Hillebrands, Enhancement of the spin pumping efficiency by spin wave mode selection, Applied Physics Letters 97 (25) (2010) 252504.

[22] S. M. Rezende, R. L. Rodriguez-Suarez, M. M. Soares, L. H. Vilela-Leao, D. L. Dominguez, A. Azevedo, Enhanced spin pumping damping in yttrium iron garnet/pt bilayers, Applied Physics Letters 102 (1) (2013) 012402. doi:10.1063/1.4773993.

[23] X. Jia, K. Liu, K. Xia, G. E. W. Bauer, Spin transfer torque on magnetic insulators, EPL (Europhysics Letters) 96 (1) (2011) 17005.

[24] A. Kapelrud, A. Brataas, Spin-pumping and enhanced gilbert damping in thin magnetic insulator films, arXiv:1303.4922.

[25] H. Puszkarski, Surface modes in magnetic thin films and in spin wave resonance, IEEE Transactions on Magnetics 9 (1) (1973) 22-27.

[26] P. Wigen, Microwave properties of magnetic garnet thin films, Thin Solid Films 114 (1-2) (1984) 135186.

[27] C. E. Patton, Magnetic excitations in solids, Physics Reports 103 (5) (1984) 251-315.

[28] G. L. da Silva, L. H. Vilela-Leo, S. M. Rezende, A. Azevedo, Enhancement of spin wave excitation by spin currents due to thermal gradient and spin pumping in yttrium iron garnet/Pt, Applied Physics Letters 102 (1) (2013) 012401-012401-4. 